\title{
Development and validation of a prognostic nomogram for predicting overall survival in patients with primary bladder sarcoma: a SEER-based retrospective study
}

Shijie Li, Xuefeng Liu and Xiaonan Chen*

\begin{abstract}
Background: Primary bladder sarcoma (PBS) is a rare malignant tumor of the bladder with a poor prognosis, and its disease course is inadequately understood. Therefore, our study aimed to establish a prognostic model to determine individualized prognosis of patients with PBS.

Patients and Methods: Data of 866 patients with PBS, registered from 1973 to 2015, were extracted from the surveillance, epidemiology, and end result (SEER) database. The patients included were randomly split into a training $(n=608)$ and a validation set $(n=258)$. Univariate and multivariate Cox regression analyses were employed to identify the important independent prognostic factors. A nomogram was then established to predict overall survival (OS). Using calibration curves, receiver operating characteristic curves, concordance index (C-index), decision curve analysis (DCA), net reclassification improvement (NRI) and integrated discrimination improvement (IDI), the performance of the nomogram was internally validated. We compared the nomogram with the TNM staging system. The application of the risk stratification system was tested using Kaplan-Meier survival analysis.
\end{abstract}

Results: Age at diagnosis, T-stage, N-stage, M-stage, and tumor size were identified as independent predictors of OS. C-index of the training cohort were $0.675,0.670,0.671$ for 1-, 3- and 5-year OS, respectively. And that in the validation cohort were $0.701,0.684,0.679$, respectively. Calibration curves also showed great prediction accuracy. In comparison with TNM staging system, improved net benefits in DCA, evaluated NRI and IDI were obtained. The risk stratification system can significantly distinguish the patients with different survival risk.

Conclusion: A prognostic nomogram was developed and validated in the present study to predict the prognosis of the PBS patients. It may assist clinicians in evaluating the risk factors of patients and formulating an optimal individualized treatment strategy.

Keywords: Primary bladder sarcoma, Nomogram, Prognosis, Survival analysis

*Correspondence: chenxn@cmu.edu.cn

Department of Urology, Shengjing Hospital of China Medical University,

36 Sanhao Street, Shenyang 110004, Liaoning, People's Republic of China

\section{Introduction}

Primary bladder sarcoma (PBS) is a very rare malignant tumor, accounting for less than $0.5 \%$ of all bladder tumors. The 5 -year survival rate is $10-35 \%$ [1]. Some subtypes of PBS show a high tendency toward distant metastasis and are associated with a shorter survival [2]. Published studies on PBS are scarce all over the world, original author(s) and the source, provide a link to the Creative Commons licence, and indicate if changes were made. The images or other third party material in this article are included in the article's Creative Commons licence, unless indicated otherwise in a credit line to the material. If material is not included in the article's Creative Commons licence and your intended use is not permitted by statutory regulation or exceeds the permitted use, you will need to obtain permission directly from the copyright holder. To view a copy of this licence, visit http://creativecommons.org/licenses/by/4.0/. The Creative Commons Public Domain Dedication waiver (http://creativecommons.org/publicdomain/zero/1.0/) applies to the data made available in this article, unless otherwise stated in a credit line to the data. 
and most of the cases are reported in the form of case reports for a certain subtype of PBS [3-5].

In view of the scarcity of PBS, the natural history of this disease is not well known. Nevertheless, its relationship with schistosomiasis [6], cyclophosphamide therapy [7, 8], and radiotherapy [9] has been documented. Leiomyosarcoma is the most common type of bladder sarcoma in adults and it is reported that the incidence rate of bladder leiomyosarcoma may increase due to an increase in the number of patients undergoing chemo or radiotherapy [10].

Insufficient understanding makes the diagnosis and treatment of PBS challenging in daily practice. Due to the low incidence rate, the treatment of PBS is largely empirical. Radical cystectomy remains the mainstay of treatment for non-metastatic PBS, and bilateral pelvic lymph node dissection is recommended because of the high risk of metastasis to the pelvic lymph nodes [11]. Transurethral resection alone is generally not recommended, except for very small lesions [3]. Chemo and radiotherapy are viable treatment options as well, and are usually used in the comprehensive treatment of rhabdomyosarcoma or high-grade metastatic leiomyosarcoma $[12,13]$. Radiotherapy is also chosen in case of positive surgical margins or suspicion of residual tumor [14].

For rare tumors such as PBS, single center studies often have poor predictive power due to the small number of patients. Therefore, using a population-based cancer database to assess the clinical characteristics and prognosis is a reasonable way to acquire better understanding of this rare disease. In this study, we used the Surveillance, Epidemiology, and End Results (SEER) database (https:// seer.cancer.gov/) to identify the prognostic factors and construct a nomogram for PBS patients. To the best of our knowledge, this is the first study using the SEER database to examine the clinical features of PBS. This study aimed to establish a predictive model to better understand the survival outcomes of PBS at a population level.

\section{Material and methods \\ Patients}

Data of the patients diagnosed with bladder sarcoma were extracted based on the International Classification of Tumor Diseases Third Edition (ICD-O-3). Inclusion criteria were as follows: (1) age at diagnosis $>18$ years old; (2) histologically diagnosed as the first malignant tumor; (3) availability of complete demographic and sociological information, follow-up date, duration of survival (in months), and cause of death; (4) histological diagnosis of bladder sarcoma (ICD-O-3 Code: C67.0-C67.9); (5) adequate data regarding the patients' clinical stage, pathological grade, and other variables. After screening, 866 eligible PBS patients were finally included in the cohort. The process of data selection was shown in Fig. 1. The patients were randomly divided into two sets (training set, $\mathrm{n}=608$ and validation set, $\mathrm{n}=258$ ). Since SEER is a

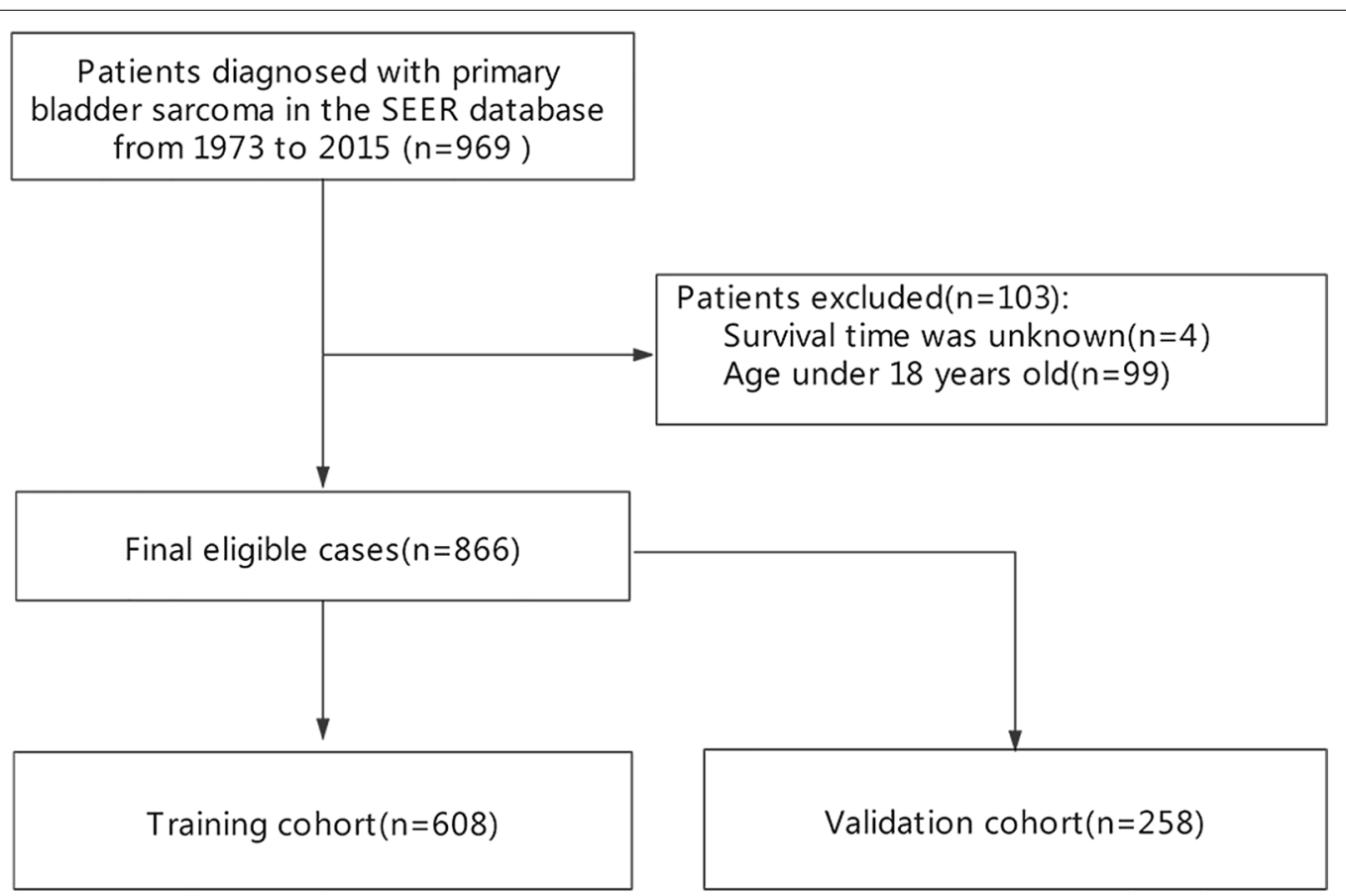

Fig. 1 Flowchart showing the selection of patients 
publicly available database, studies using the SEER database do not require ethical board approval and patient consent.

\section{Data collection}

Variables in the present study included age, sex, race, marital status, histological grade, pathological classification, pathological stage (TNM stage according to the American Joint Committee on Cancer staging system, third and sixth edition), tumor size, type of intervention such as radiation, chemotherapy and/or surgery, vital status, and duration of survival. As is shown in Fig. 2B, $\mathrm{C}$, tumor size was divided into three categories by X-tile software version 3.6.1 (Rimm Lab, Yale School of Medicine, New Haven, CT, USA), which is a useful tool for finding optimal cutoff points of continuous data [15]. Overall survival (OS) was the primary endpoint. Duration of survival was calculated from the date of diagnosis to the date of last follow-up or until the date of death.

\section{Statistical analysis}

Continuous variables were reported as median with range, and categorical variables as frequencies and proportions. The optimal cutoff values for age and tumor size were evaluated using the X-tile software. Univariate Cox regression analysis was performed to identify the significant prognostic factors. Afterwards, we incorporated them into the multivariable Cox proportional hazards regression models to further determine each variable's independent association with survival outcomes. A nomogram for predicting the 1-, 3- and 5-year OS was constructed using the factors which remained significant in the multivariate Cox regression model. The predictive accuracy and discriminative ability of the nomogram were determined by the receiver operating characteristics (ROC) curves, the area under the curve (AUC), and Harrell's concordance index (C-index). Calibration plots were generated to explore the performance characteristics of the nomogram at 1-, 3- and 5-year survival time. In addition, decision curve analysis (DCA), net reclassification improvement (NRI) and integrated discrimination improvement (IDI) were used to evaluate the clinical utility of the nomogram and to assess whether the model was more accurate than AJCC TNM staging system or not.

In addition, we calculated the total score of each patient based on the nomogram and constructed a risk stratification model accordingly, dividing the cohort into

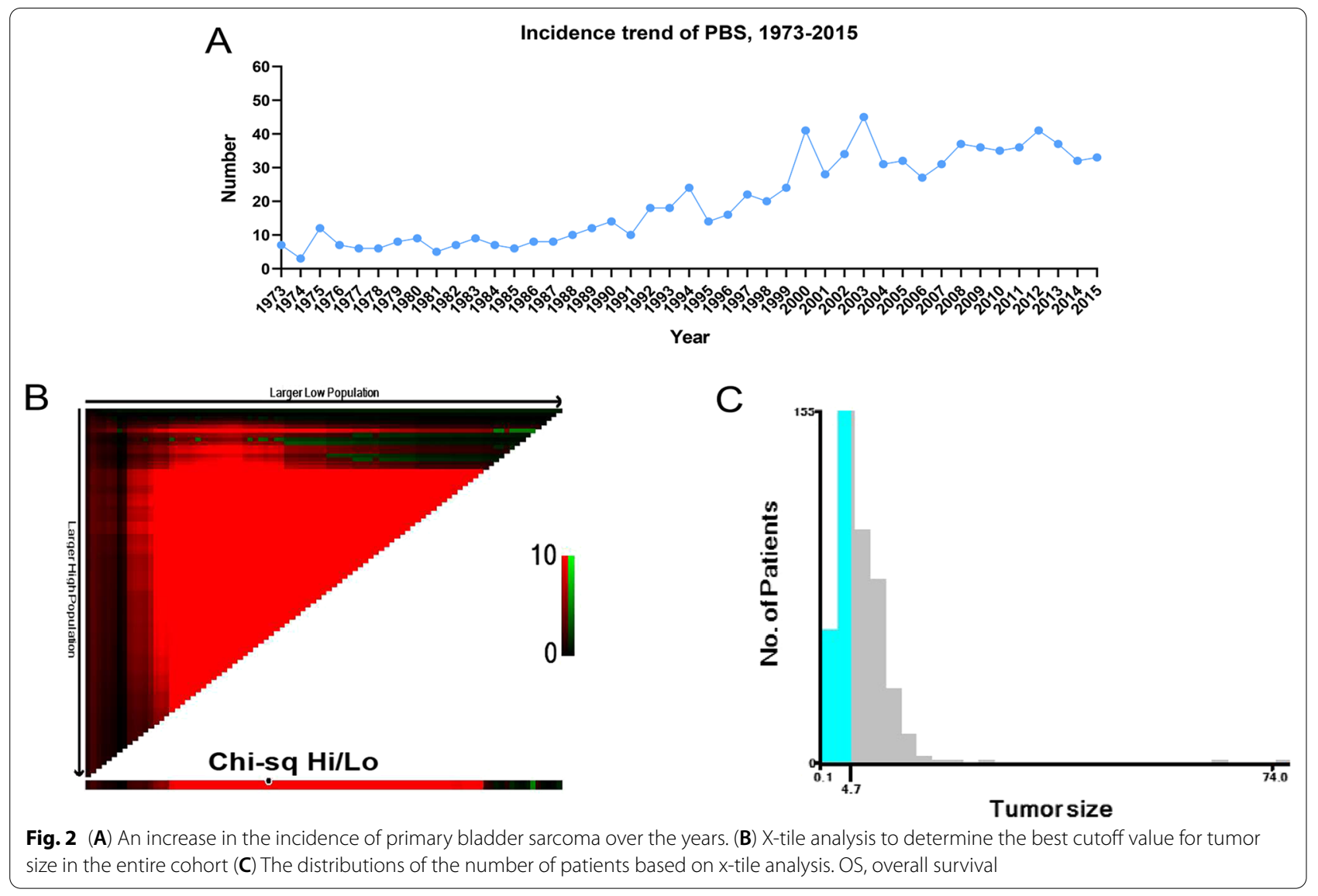


Table 1 Baseline demographic and clinical characteristics of patients with primary bladder sarcoma in the training cohort and validation cohort

\begin{tabular}{|c|c|c|c|c|}
\hline Characteristics & Total cohort $(n=866)$ & Training cohort $(n=608)$ & $\begin{array}{l}\text { Validation cohort } \\
(\mathrm{n}=258)\end{array}$ & $P$-value \\
\hline Total & 866 & 608 & 258 & \\
\hline Age (IQR) & $73.0(60.2,81.0)$ & $73.0(60.8,81.0)$ & $73.0(60.2,81.8)$ & 0.963 \\
\hline Age, $n(\%)$ & & & & 0.517 \\
\hline $18-29$ & $27(3.1)$ & $21(3.5)$ & $6(2.3)$ & \\
\hline $30-39$ & $28(3.2)$ & $22(3.6)$ & $6(2.3)$ & \\
\hline $40-49$ & $56(6.5)$ & $33(5.4)$ & $23(8.9)$ & \\
\hline $50-59$ & $94(10.9)$ & $67(11)$ & $27(10.5)$ & \\
\hline $60-69$ & $156(18.0)$ & $109(17.9)$ & $47(18.2)$ & \\
\hline $70-79$ & $248(28.6)$ & $176(28.9)$ & $72(27.9)$ & \\
\hline$\geq 80$ & $257(29.7)$ & $180(29.6)$ & $77(29.8)$ & \\
\hline $\operatorname{Sex}, n(\%)$ & & & & 1.000 \\
\hline Female & $337(38.9)$ & $237(39)$ & $100(38.8)$ & \\
\hline Male & $529(61.1)$ & $371(61)$ & $158(61.2)$ & \\
\hline Race, $n(\%)$ & & & & 0.189 \\
\hline White & $743(85.8)$ & $530(87.2)$ & $213(82.6)$ & \\
\hline Black & $90(10.4)$ & $58(9.5)$ & $32(12.4)$ & \\
\hline Other & $33(3.8)$ & $20(3.3)$ & $13(5)$ & \\
\hline Marital status, n (\%) & & & & 0.381 \\
\hline Married & $484(55.9)$ & $342(56.2)$ & $142(55)$ & \\
\hline Single & $353(40.8)$ & $249(41)$ & $104(40.3)$ & \\
\hline Unknown & $29(3.3)$ & $17(2.8)$ & $12(4.7)$ & \\
\hline Pathological classification, n (\%) & & & 0.633 & \\
\hline Carcinosarcoma & $408(47.1)$ & $296(48.7)$ & $112(43.4)$ & \\
\hline Leiomyosarcoma & $207(23.9)$ & $141(23.2)$ & $66(25.6)$ & \\
\hline Sarcoma & $84(9.7)$ & $59(9.7)$ & $25(9.7)$ & \\
\hline Spindle cell sarcoma & $31(3.6)$ & $22(3.6)$ & $9(3.5)$ & \\
\hline Other & $136(15.7)$ & $90(14.8)$ & $46(17.8)$ & \\
\hline Histological grade, n (\%) & & & & 0.166 \\
\hline Well differentiated & $22(2.5)$ & $16(2.6)$ & $6(2.3)$ & \\
\hline Moderately differentiated & $38(4.4)$ & $29(4.8)$ & $9(3.5)$ & \\
\hline Poorly differentiated & $202(23.3)$ & $151(24.8)$ & $51(19.8)$ & \\
\hline Undifferentiated & $283(32.7)$ & $184(30.3)$ & $99(38.4)$ & \\
\hline Unknown & $321(37.1)$ & $228(37.5)$ & $93(36)$ & \\
\hline T-stage, $n(\%)$ & & & & 0.124 \\
\hline $\mathrm{Ta}$ & $68(7.9)$ & $55(9)$ & $13(5)$ & \\
\hline Tis & $1(0.1)$ & $1(0.2)$ & $0(0)$ & \\
\hline T1 & $68(7.9)$ & $45(7.4)$ & $23(8.9)$ & \\
\hline $\mathrm{T} 2$ & $94(10.9)$ & $63(10.4)$ & $31(12)$ & \\
\hline T3 & $92(10.6)$ & $72(11.8)$ & $20(7.8)$ & \\
\hline $\mathrm{T} 4$ & $54(6.2)$ & $40(6.6)$ & $14(5.4)$ & \\
\hline Unknown & $489(56.5)$ & $332(54.6)$ & $157(60.9)$ & \\
\hline$N$-stage, n (\%) & & & & 0.286 \\
\hline No & $476(55.0)$ & $329(54.1)$ & $147(57)$ & \\
\hline Yes & $175(20.2)$ & 119 (19.6) & $56(21.7)$ & \\
\hline Unknown & $215(24.8)$ & $160(26.3)$ & $55(21.3)$ & \\
\hline M-stage, n (\%) & & & & 0.326 \\
\hline No & $593(68.5)$ & 407 (66.9) & $186(72.1)$ & \\
\hline Yes & $97(11.2)$ & $71(11.7)$ & $26(10.1)$ & \\
\hline
\end{tabular}


Table 1 (continued)

\begin{tabular}{|c|c|c|c|c|}
\hline Characteristics & Total cohort $(\mathrm{n}=\mathbf{8 6 6})$ & Training cohort $(n=608)$ & $\begin{array}{l}\text { Validation cohort } \\
(n=258)\end{array}$ & $P$-value \\
\hline Unknown & $176(20.3)$ & $130(21.4)$ & $46(17.8)$ & \\
\hline Tumor size, n (\%) & & & & 0.622 \\
\hline$<4.8$ & 161 (18.6) & $117(19.2)$ & $44(17.1)$ & \\
\hline$\geq 4.8$ & 291 (33.6) & $199(32.7)$ & $92(35.7)$ & \\
\hline Unknown & $414(47.8)$ & $292(48)$ & $122(47.3)$ & \\
\hline Surgical treatment, $n(\%)$ & & & & $0.023^{*}$ \\
\hline No & $54(6.2)$ & $38(6.2)$ & $16(6.2)$ & \\
\hline Yes & $537(62.0)$ & $360(59.2)$ & $177(68.6)$ & \\
\hline Unknown & $275(31.8)$ & $210(34.5)$ & $65(25.2)$ & \\
\hline Radiation & & & & 0.360 \\
\hline No/Unknown, n (\%) & $754(87.1)$ & $534(87.8)$ & $220(85.3)$ & \\
\hline Yes & $112(12.9)$ & $74(12.2)$ & $38(14.7)$ & \\
\hline Chemotherapy, n (\%) & & & & 0.864 \\
\hline No/Unknown & $734(84.8)$ & $514(84.5)$ & $220(85.3)$ & \\
\hline Yes & $132(15.2)$ & $94(15.5)$ & $38(14.7)$ & \\
\hline
\end{tabular}

${ }^{*} P<0.05$ indicating statistical significance

two different risk groups (low-risk group and high-risk group). The optimal cutoff value was analyzed by X-tile software. Kaplan-Meier survival analysis and Chi-square test were used to assess the significance of the difference in survival between the low- and high-risk groups.

All the analyses were performed with the statistical software package R 4.0.2 (http://www.R-project.org, The R Foundation, Vienna, Austria). Two-sided $P$ values of less than 0.05 were considered statistically significant. All procedures performed in this study involving human participants conformed to the ethical standards described in the 1964 Helsinki declaration and its subsequent amendments.

\section{Results}

\section{Patients' baseline characteristics}

Eight hundred and sixty-six patients with PBS in the SEER database met the study criteria and were included in this study. Figure 2A clearly showed an increase in the incidence of PBS over the years. Cohort demographics and tumor-related characteristics were described in Table 1. Most patients were male $(529,61.1 \%)$, white (743, 85.8\%), and more than 80 years old $(257,29.7 \%)$. With regard to therapy, a majority of the patients underwent surgery $(537,62.0 \%)$, while fewer patients received radiation $(112,12.9 \%)$ or chemotherapy $(132,15.2 \%)$. Overall, the 1-, 3- and 5-year OS rates were 48.2\%, 34.5\% and $29.4 \%$, respectively. Except for surgical treatment, there was no significant difference between the training and validation set $(P>0.05)$.
There were 709 events (deaths) in the cohort and the mean follow-up period was 42.1 months (median, 9.0 months; range $0-447$ months).

\section{Screening for prognostic factors of OS}

We conducted a univariable and multivariable Cox proportional hazards regression analysis to demonstrate the association between selected characteristics and oncological outcomes. Univariable Cox regression analysis identified seven variables (age, pathological classification, T-stage, $\mathrm{N}$-stage, $\mathrm{M}$-stage, tumor size, and radiation) as factors associated with a shorter OS. Multivariable Cox regression analysis indicated that statistically significant risk factors associated with a shorter OS included age, T-stage, $\mathrm{N}$-stage, M-stage, and tumor size (Table 2). For example, patients with a tumor of a higher $\mathrm{T}$ stage or distant metastases may have a poor prognosis and worse cancer outcomes. Similarly, patients with a large tumor $(\geq 4.8 \mathrm{~cm})$ were more likely to have poor prognosis. Median OS in the training cohort was 11 months, with 1-, 3- and 5-year survival rates of $51.3 \%, 36.2 \%$, and $30.8 \%$, respectively. Kaplan-Meier analysis intuitively showed the different survival outcomes stratified according to the variables listed in Table 1 (Fig. 3A-M). Log-rank test showed significant differences in OS among subgroups in terms of pathological classification, T-stage, $\mathrm{N}$-stage, M-stage, tumor size and radiotherapy $(P<0.05)$. 
Table 2 Univariate and multivariate Cox regression analysis of included variables for OS in training cohort

\begin{tabular}{|c|c|c|c|c|}
\hline \multirow[t]{2}{*}{ Characteristics } & \multicolumn{2}{|l|}{ Univariate analysis } & \multicolumn{2}{|c|}{ Multivariate analysis } \\
\hline & HR $(95 \% \mathrm{Cl})$ & $P$-value & HR $(95 \% \mathrm{Cl})$ & $P$-value \\
\hline \multicolumn{5}{|l|}{ Age } \\
\hline $18-29$ & Reference & & Reference & \\
\hline $30-39$ & $0.53(0.27,1.03)$ & 0.06 & $0.48(0.24,0.96)$ & $0.039 *$ \\
\hline $40-49$ & $0.56(0.31,1.03)$ & 0.062 & $0.43(0.23,0.80)$ & $0.008^{*}$ \\
\hline $50-59$ & $0.71(0.41,1.2)$ & 0.202 & $0.74(0.42,1.27)$ & 0.274 \\
\hline $60-69$ & $0.58(0.35,0.97)$ & $0.039^{*}$ & $0.55(0.32,0.94)$ & $0.030^{*}$ \\
\hline 70-79 & $0.65(0.4,1.06)$ & 0.081 & $0.62(0.37,1.03)$ & 0.063 \\
\hline$\geq 80$ & $0.66(0.4,1.07)$ & 0.094 & $0.61(0.37,1.03)$ & 0.063 \\
\hline \multicolumn{5}{|l|}{ Sex } \\
\hline Female & Reference & & & \\
\hline Male & $1.1(0.92,1.32)$ & 0.308 & & \\
\hline \multicolumn{5}{|l|}{ Race } \\
\hline White & Reference & & & \\
\hline Black & $1.07(0.79,1.46)$ & 0.657 & & \\
\hline Other & $1.11(0.68,1.8)$ & 0.681 & & \\
\hline \multicolumn{5}{|l|}{ Marital status } \\
\hline Married & Reference & & & \\
\hline Single & $0.9(0.75,1.08)$ & 0.275 & & \\
\hline Unknown & $1.07(0.61,1.87)$ & 0.806 & & \\
\hline \multicolumn{5}{|l|}{ Pathological classification } \\
\hline Carcinosarcoma & Reference & & Reference & \\
\hline Leiomyosarcoma & $0.99(0.79,1.25)$ & 0.927 & $0.79(0.61,1.03)$ & 0.086 \\
\hline Sarcoma & $1.55(1.16,2.07)$ & $0.003^{*}$ & $1.36(0.99,1.88)$ & 0.060 \\
\hline Spindle cell sarcoma & $1.14(0.7,1.84)$ & 0.602 & $0.94(0.56,1.60)$ & 0.829 \\
\hline Other & $1.3(1,1.69)$ & 0.051 & $1.10(0.83,1.46)$ & 0.510 \\
\hline \multicolumn{5}{|l|}{ Histological grade } \\
\hline Well differentiated & Reference & & & \\
\hline Moderately differentiated & $1.35(0.71,2.57)$ & 0.355 & & \\
\hline Poorly differentiated & $1.19(0.69,2.07)$ & 0.534 & & \\
\hline Undifferentiated & $1.28(0.74,2.21)$ & 0.381 & & \\
\hline Unknown & $1.25(0.72,2.15)$ & 0.426 & & \\
\hline \multicolumn{5}{|l|}{ T-stage } \\
\hline Ta & Reference & & Reference & \\
\hline Tis & $15.53(2.11,114.23)$ & $0.007^{*}$ & $40.68(5.17,319.99)$ & $<0.001^{*}$ \\
\hline $\mathrm{T} 1$ & $2.08(1.35,3.21)$ & $<0.001^{*}$ & $2.20(1.29,3.75)$ & $0.004^{*}$ \\
\hline $\mathrm{T} 2$ & $2.18(1.48,3.22)$ & $<0.001^{*}$ & $2.07(1.33,3.23)$ & $0.001^{*}$ \\
\hline T3 & $1.0051(0.65,1.57)$ & 0.982 & $1.15(0.68,1.96)$ & 0.595 \\
\hline $\mathrm{T} 4$ & $1.02(0.64,1.62)$ & 0.926 & $1.17(0.67,2.06)$ & 0.574 \\
\hline Unknown & $1.86(1.36,2.56)$ & $<0.001^{*}$ & $2.17(1.45,3.25)$ & $<0.001^{*}$ \\
\hline \multicolumn{5}{|l|}{$N$-stage } \\
\hline No & Reference & & Reference & \\
\hline Yes & $0.62(0.48,0.8)$ & $<0.001^{*}$ & $0.74(0.56,0.97)$ & $0.028^{*}$ \\
\hline Unknown & $0.83(0.68,1.02)$ & 0.084 & $1.03(0.71,1.50)$ & 0.869 \\
\hline \multicolumn{5}{|l|}{ M-stage } \\
\hline No & Reference & & Reference & \\
\hline Yes & $2.7(2.05,3.55)$ & $<0.001^{*}$ & $2.74(2.00,3.75)$ & $<0.001^{*}$ \\
\hline Unknown & $0.87(0.69,1.08)$ & 0.205 & $0.85(0.62,1.19)$ & 0.360 \\
\hline
\end{tabular}


Table 2 (continued)

\begin{tabular}{|c|c|c|c|c|}
\hline \multirow[t]{2}{*}{ Characteristics } & \multicolumn{2}{|c|}{ Univariate analysis } & \multicolumn{2}{|c|}{ Multivariate analysis } \\
\hline & HR $(95 \% \mathrm{Cl})$ & $P$-value & HR $(95 \% \mathrm{Cl})$ & $P$-value \\
\hline \multicolumn{5}{|l|}{ Tumor size } \\
\hline$<4.8$ & Reference & & Reference & \\
\hline$\geq 4.8$ & $2(1.51,2.63)$ & $<0.001^{*}$ & $2.02(1.52,2.69)$ & $<0.001^{*}$ \\
\hline Unknown & $1.86(1.44,2.41)$ & $<0.001^{*}$ & $2.11(1.59,2.81)$ & $<0.001^{*}$ \\
\hline \multicolumn{5}{|l|}{ Surgical treatment } \\
\hline No & Reference & & & \\
\hline Yes & $0.84(0.57,1.23)$ & 0.364 & & \\
\hline Unknown & $0.84(0.56,1.25)$ & 0.396 & & \\
\hline \multicolumn{5}{|l|}{ Radiation } \\
\hline No/Unknown & Reference & & & \\
\hline Yes & $1.39(1.06,1.81)$ & $0.017^{*}$ & $1.26(0.94,1.70)$ & 0.118 \\
\hline \multicolumn{5}{|l|}{ Chemotherapy } \\
\hline No/Unknown & Reference & & & \\
\hline Yes & $0.97(0.75,1.26)$ & 0.842 & & \\
\hline
\end{tabular}

${ }^{*} P<0.05$ indicating statistical significance

\section{Prognostic nomogram construction for OS}

A nomogram was established based on the aforementioned significant prognostic factors for 1-, 3- and 5-year OS (Fig. 4), and then was validated internally. Each variable was given a score based on the hazard ratio. The total scores for each variable were added up and placed on the total subscale to obtain the probabilities of 1-, 3- and 5-year OS. As shown in Additional file 1: Figure S1, using the nomogram, it could be concluded that a 70-year-old patient with T2N0M0 and a tumor size of $5 \mathrm{~cm}$ would score 47.5 points, which means that the patient has about $57.5 \%, 42 \%$, and $34.5 \%$ survival probability 1,3 , and 5 years after the diagnosis, respectively.

\section{Calibration and validation of the nomogram}

On the training cohort, the $\mathrm{C}$-index of the nomogram for 1-, 3- and 5-year OS prediction were 0.675 [95\% confidence interval (CI): 0.648-0.702], 0.670 (95\% CI: 0.6420.697 ) and 0.671 (95\% CI: 0.643-0.698), respectively. On the validation cohort, the $\mathrm{C}$-indexes at 1-, 3-, and 5-year were 0.701 (95\% CI 0.674-0.728), 0.684 (95\% CI 0.6570.711 ), and 0.679 (95\% CI $0.651-0.706)$, respectively. The data indicated brilliant discrimination ability of the nomogram.

Meanwhile, the calibration plots of the training cohort for 1-, 3- and 5-year OS displayed consistency between the observed and predicted results (Fig. 5A-C). Similarly, the calibration plots of the 1-, 3- and 5-year OS were well calibrated in the validation cohort (Fig. 5D-F).

\section{Comparison of the nomogram and AJCC TNM staging system}

ROC curves analysis showed that the AUCs of the nomogram for 1-, 3- and 5-year OS were better than those of TNM stage both in the training (Fig. 6A-C) and validation cohort (Fig. 6D-F).

DCA analysis showed that compared with the AJCC TNM staging system, the net benefit of the new nomogram is significantly increased and has a wide range of threshold probabilities both in the training (Fig. 7A-C) and validation cohort (Fig. 7D-F). This indicated that the nomogram can be more beneficial in the clinical application of predicting individual survival outcomes than TNM staging system.

In the NRI and IDI analyses, the nomogram performed better than TNM staging system (Table 3). In the training cohort, the 1-, 3- and 5-year NRI of the nomogram compared to TNM staging system was $15.3 \%(p<0.01), 21.0 \%$ $(p<0.01)$ and $21.2 \%(p<0.01)$, respectively. And the $1-, 3$ and 5-year IDI of the nomogram compared to TNM staging system was $3.3 \%(p<0.01), 4.6 \%(p<0.01)$ and $4.4 \%$ $(p<0.01)$, respectively. In the validation cohort, the 1-, 3 - and 5-year NRI of the nomogram compared to TNM staging system was $19.8 \%(p<0.01), 16.5 \%(p=0.02)$ and $17.9 \%(p=0.01)$, respectively. And the 1-, 3- and 5-year IDI of the nomogram compared to TNM staging system was $7.9 \%(p<0.01), 7.9 \%(p<0.01)$ and $8.6 \%(p<0.01)$, respectively. 


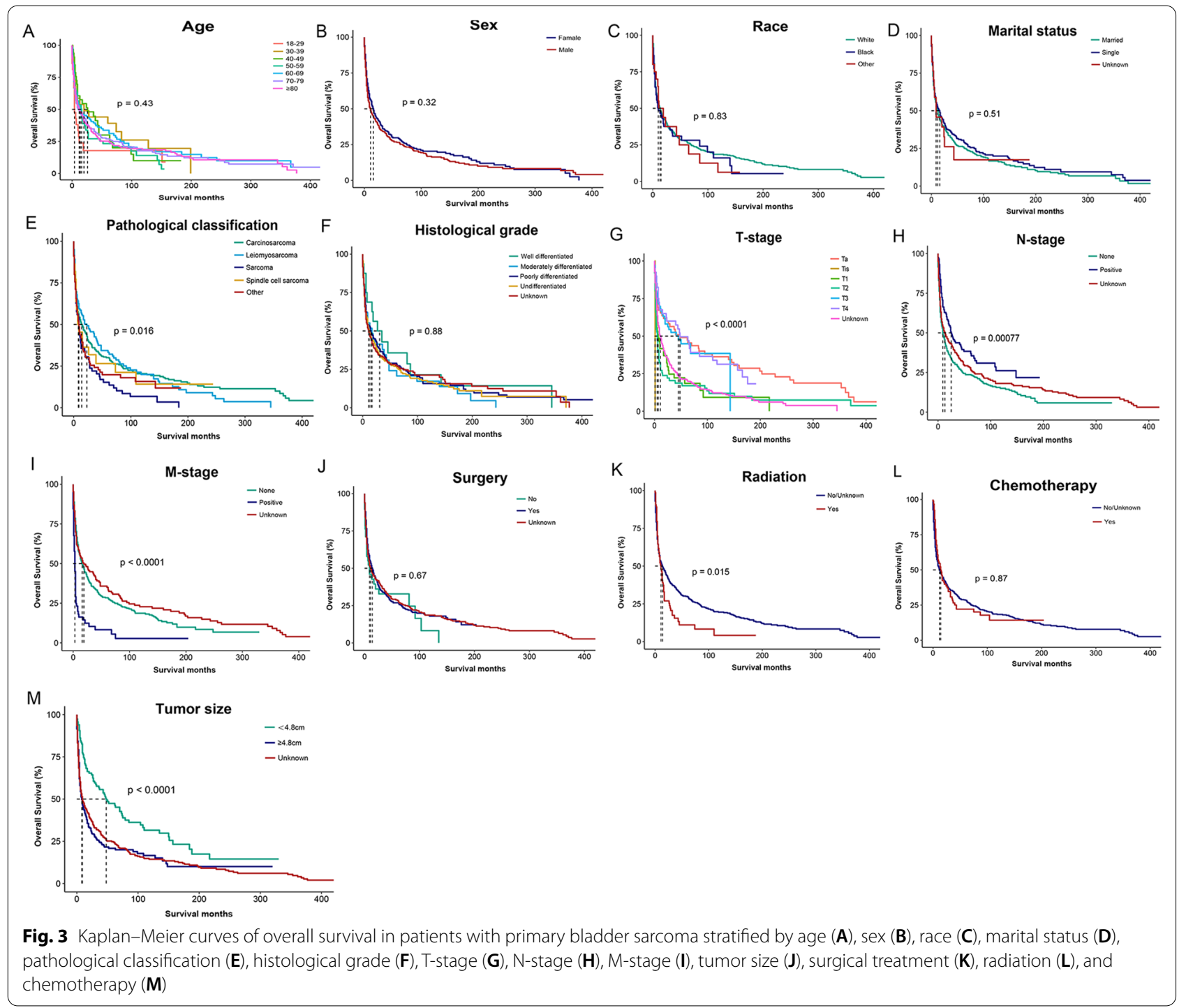

\section{Ability of nomogram to stratify patient risk}

The cut-off point between the high-risk and low-risk cohorts was determined as 47 by $\mathrm{X}$-tile analysis, and the 608 patients in the training cohort were divided into a high-risk group (total score $>47$ ) and a low-risk group (total score $\leq 47$ ) based on this cut-off value. By KaplanMeier analysis (Fig. 8A), 367 high-risk patients had significantly more severe OS than 241 low-risk patients $(p<0.0001)$. Application of this cutoff value also significantly distinguished the high-risk and low-risk groups in the validation cohort $(p=0.041)$ (Fig. 8B).

\section{Discussion}

Around 430,000 new cases of bladder cancer are diagnosed worldwide each year, and this cancer is associated with high morbidity and mortality [16]. As a rare subtype of bladder cancer, the incidence rate of PBS is very low, due to which tumor progression is not well-understood. The clinical significance and biologic behavior of this subtype of bladder cancer warrant additional investigation. In the present study, vast amount of data collected by the SEER program was utilized to examine the largest series of PBS cases reported to date. This study was the first attempt to date to use the SEER database to build a predictive model for better understanding the survival outcomes of PBS at a population level.

Several unique features of PBS distinguish it from urothelial bladder cancer, and are worth mentioning. PBS has multiple types, and leiomyosarcoma and rhabdomyosarcoma account for $50 \%$ and $20 \%$ of PBS cases, respectively [17]. Other histological types of PBS include osteosarcoma, angiosarcoma, myxoid liposarcoma, fibrosarcoma, malignant fibroblastic tumor, carcinosarcoma, and plexiform sarcoma [18]. Besides, previous studies 


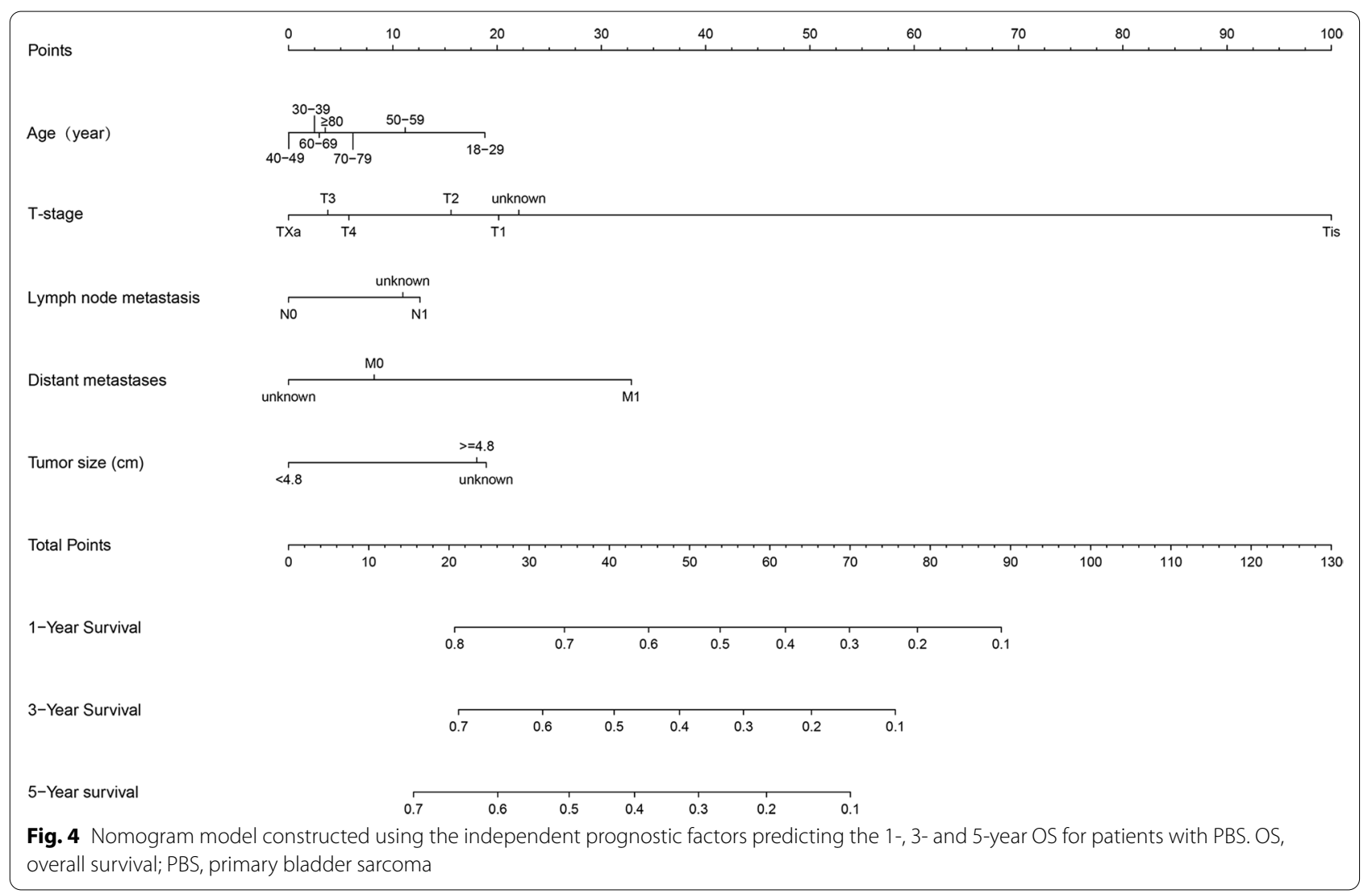
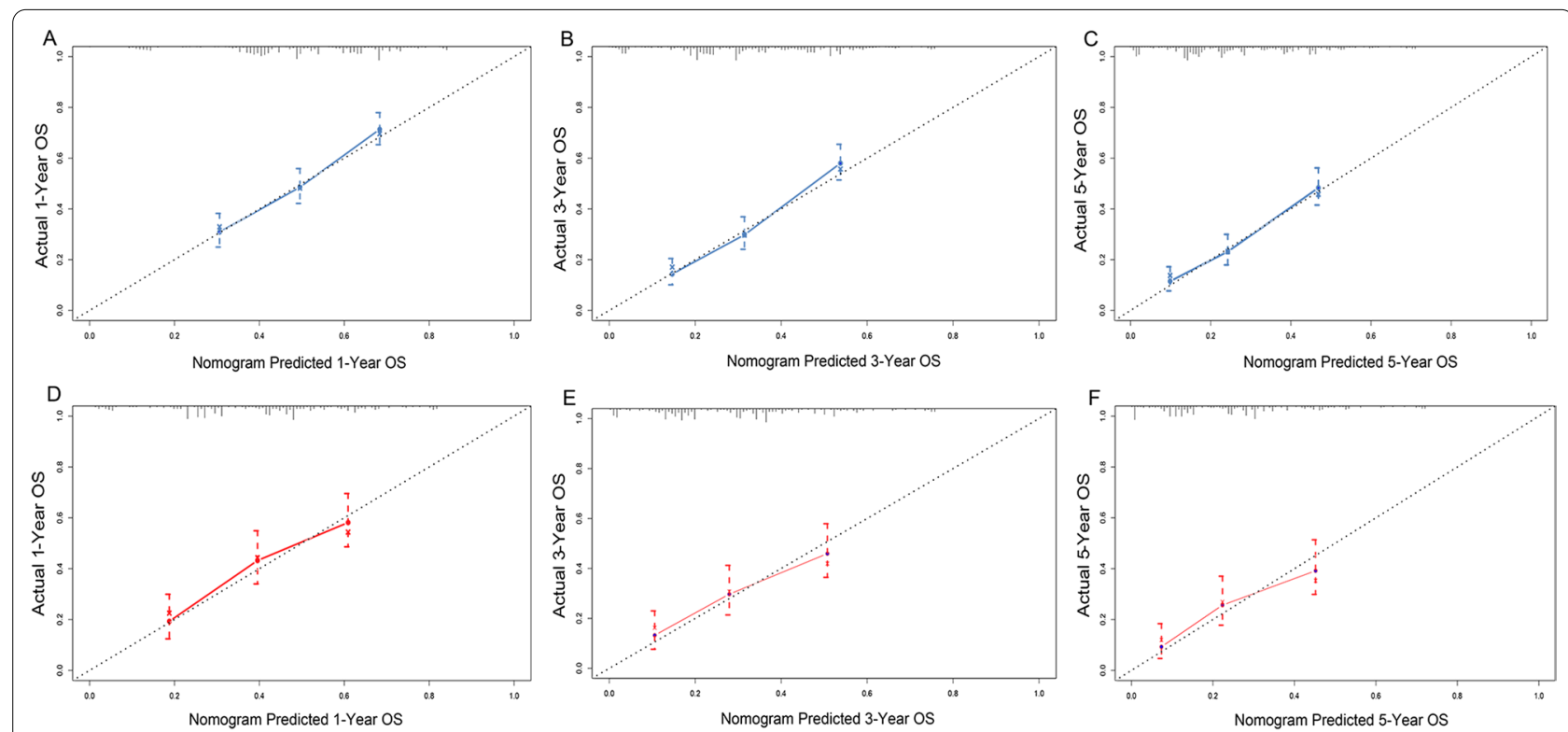

Fig. 5 Calibration plots for the nomogram. Calibration plots of 1-year (A), 3-year (B), and 5-year (C) OS in the training cohort; Calibration plots of 1-year (D), 3-year (E), and 5-year (F) OS in the validation cohort. OS, overall survival 

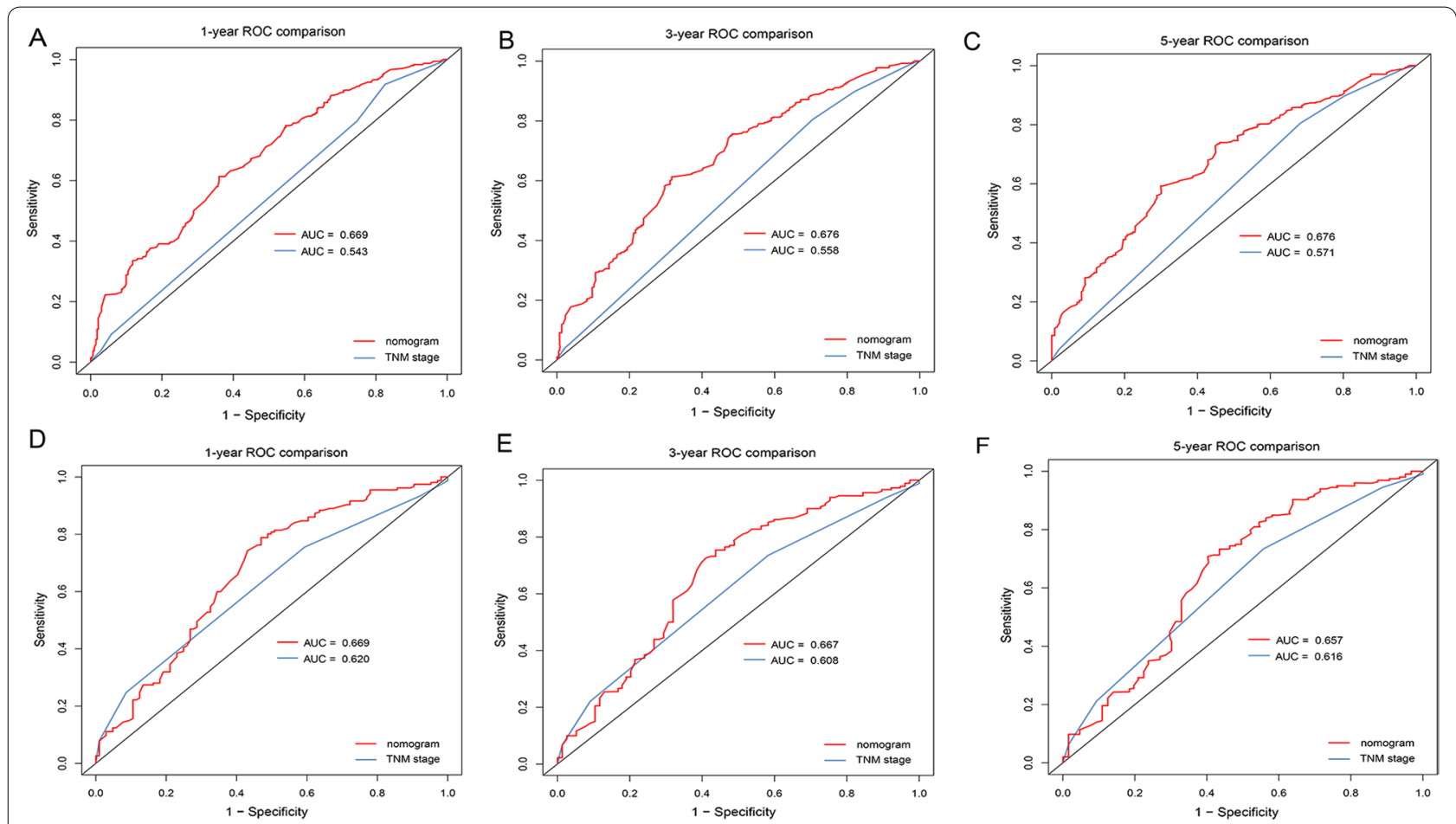

Fig. 6 ROC curves of the nomogram for OS compared with TNM staging. ROC curves comparation of the nomogram and TNM staging for 1-year (A), 3-year (B) and 5-year (C) OS in the training cohort. ROC curves comparation of the nomogram and TNM staging for 1-year (D), 3-year (E) and 5-year (F) OS in the validation cohort. AUC: area under the curve; ROC, receiver operating characteristic; OS, overall survival

have emphasized the poor prognosis of PBS. Rosser et al. [19] reported one of the largest series of 36 adult PBS patients treated between 1986 and 1998. The 5-year disease-specific survival rate was $62.0 \%$. In another systematic review and meta-analysis that included by far the largest number of cases containing 210 patients with PBS between 1970 and 2018, Zieschang et al. [20] determined a 5-year cancer-specific cumulative mortality rate of $38 \%$ for patients with PBS. We found that the prognosis of PBS was poor and did not change significantly over decades. In addition, previous studies have shown that the vast majority of PBS patients are elderly men with significant pain. However, hematuria is rare, which is very different from the presentation of urothelial tumors [21, 22]. The treatment of this rare tumor was challenging. The most promising treatment options still seemed to be radical cystectomy over the past few decades, possibly supplemented by chemotherapy or radiotherapy. However, as time migrated and technology developed, partial cystectomy was one of the surgical options available for smaller tumors.

To date, there are no large, prospective, randomized controlled trials on PBS treatment strategies worldwide.
Therefore, it is unknown whether the survival rate of the patients depends upon the type of treatment modality. Due to the specificity of PBS, there is no specially designed or widely accepted grading system or prediction model. However, early identification of the disease and effective treatment can significantly improve the prognosis. Hence, efforts to establish predictive models to promote the management of these patients according to their individualized prognosis are justified. In our study, we established and validated a novel predictive tool based on age, tumor stage, lymph node status, distant metastasis, and tumor size that can be used to guide clinical practice.

In recent years, nomograms, developed using the SEER database, have been widely used to predict the prognosis of various malignancies, such as Ewing sarcoma, penile carcinoma, and cardiac sarcoma [23-25]. Our current study was the first to construct a well-validated nomogram including several clinical features and risk scores for patients with PBS, which can predict the clinical prognosis of PBS intuitively and effectively. The variables in the nomogram were independent factors affecting OS, which led to a better prediction of the survival of patients with 


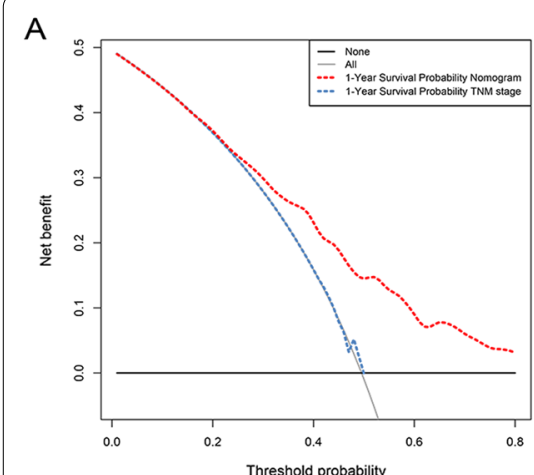

D

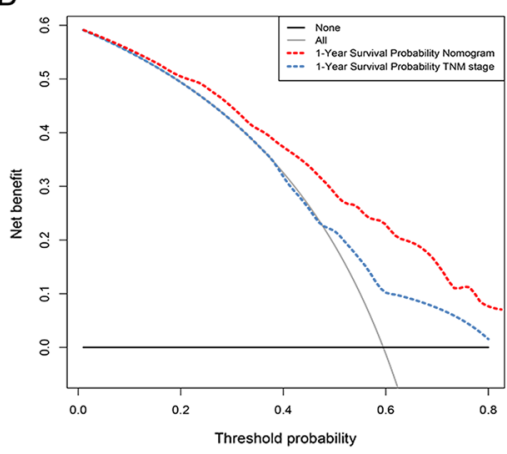

B

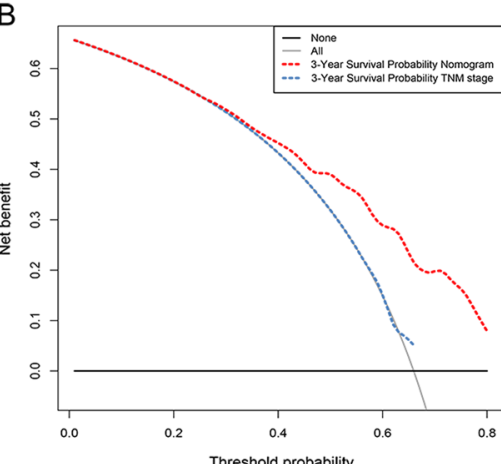

$\mathrm{E}$

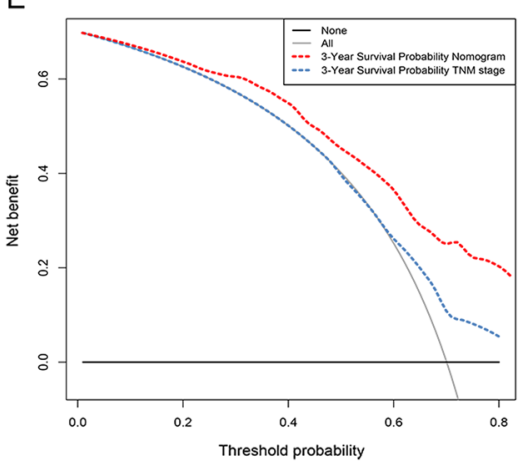

C

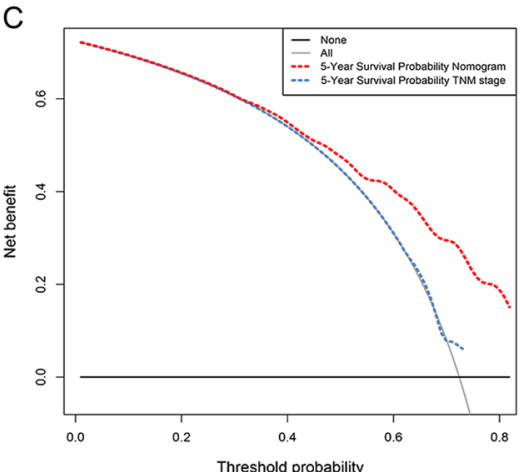

$\mathrm{F}$

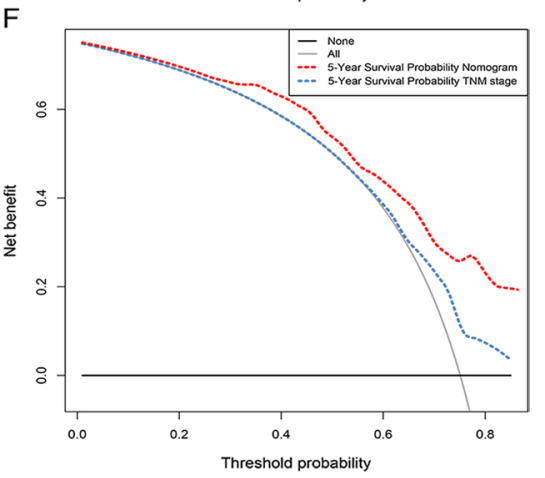

Fig. 7 DCA of the nomogram and AJCC TNM staging for 1-year (A), 3-year (B) and 5-year (C) OS in training cohort, and for 1-year (D), 3-year (E) and 5 -year (F) OS in the validation cohort. The red dashed line represents the nomogram. The blue dashed line represents AJCC TNM stage. OS, overall survival; DCA, decision curve analyses; AJCC, American Joint Committee on Cancer

Table $3 \mathrm{NRI}$ and IDI of the nomogram in survival prediction for PBS patients compared with TNM staging

\begin{tabular}{|c|c|c|c|c|c|c|}
\hline \multirow[t]{2}{*}{ Index } & \multicolumn{3}{|c|}{ Training cohort } & \multicolumn{3}{|c|}{ Validation cohort } \\
\hline & Estimate & $95 \% \mathrm{Cl}$ & $P$-value & Estimate & $95 \% \mathrm{Cl}$ & $P$-value \\
\hline \multicolumn{7}{|c|}{ NRI (vs. TNM staging) } \\
\hline For 1-year OS & 0.153 & $0.048-0.238$ & $<0.01^{*}$ & 0.198 & $0.072-0.367$ & $<0.01^{*}$ \\
\hline For 3-year OS & 0.210 & $0.084-0.297$ & $<0.01^{*}$ & 0.165 & $0.026-0.343$ & $0.02^{*}$ \\
\hline For 5-year OS & 0.212 & $0.084-0.304$ & $<0.01^{*}$ & 0.179 & $0.029-0.362$ & $0.01^{*}$ \\
\hline \multicolumn{7}{|c|}{ IDI (vs. TNM staging) } \\
\hline For 1-year OS & 0.033 & $0.015-0.058$ & $<0.01^{*}$ & 0.079 & $0.037-0.149$ & $<0.01^{*}$ \\
\hline For 3-year OS & 0.046 & $0.019-0.081$ & $<0.01^{*}$ & 0.079 & $0.033-0.151$ & $<0.01^{*}$ \\
\hline For 5-year OS & 0.044 & $0.017-0.081$ & $<0.01^{*}$ & 0.086 & $0.032-0.169$ & $<0.01^{*}$ \\
\hline
\end{tabular}

$\mathrm{NRI}$, net reclassification improvement; IDI, integrated discrimination improvement; PBS, primary bladder sarcoma; OS, overall survival

${ }^{*} P<0.05$ indicating statistical significance

PBS. Using this nomogram, we will be able to predict the future survival rate of the patients more accurately. Although the C-indexes and AUCs of the nomogram in the training and validation cohort were not high enough, the predictive ability of the model was more accurate than using the current TNM staging to predict the prognosis. Further DCA, NRI and IDI analyses demonstrated its clear clinical application advantages over the TNM staging system. A risk stratification model based on this nomogram can effectively classify patients in the training or validation cohort into two risk groups (high risk and low risk) and OS can be distinguished. The results of this study could be particularly helpful in predicting postoperative survival of the PBS patients.

Our nomogram is innovative and reasonable in the following aspects: firstly, to the best of our knowledge, 

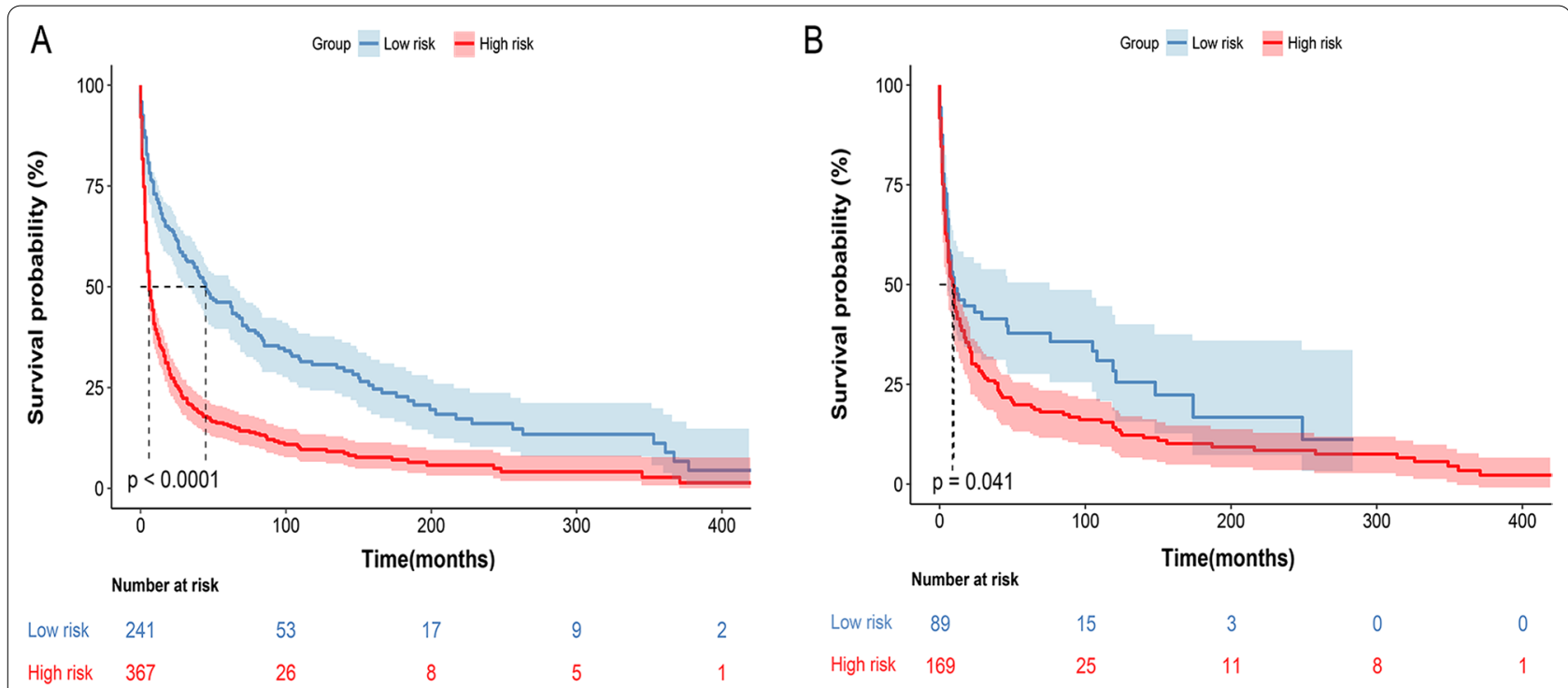

Fig. 8 Kaplan-Meier survival analyses to test the risk stratification system within the training $(\mathbf{A})$ and the validation cohort (B). The blue line represents low-risk group, and the red line represents high-risk group

this study is the first to attempt to develop a prognostic nomogram for OS of the PBS patients using populationbased data, which can provide individualized treatment guidance. Secondly, variables like age, T/N/M stage, and tumor size were used to develop this nomogram. It is worth noting that in order to maintain the integrity of the data, factors containing negative or unknown information were also included in the analysis. Taking tumor size as an example, 414 cases (47.8\%) had unknown tumor size. In addition, according to the multivariate Cox regression analysis and nomogram, unknown tumor size seemed to reduce the survival rate, which may be due to the heterogeneity of these tumors. However, we could not exclude this group of patients from the study; otherwise, potential selection bias could have been introduced. On the contrary, using continuous queues and complete information can guarantee more accurate results. Finally, the nomogram based on the SEER database was able to predict the prognosis of PBS. ROC curve, DCA, NRI and IDI analyses of this study showed that the nomogram could predict the death of patients with PBS more accurately, which has clinical applicability. The results of the internal validation of nomogram prediction were found to be consistent.

There are some limitations in our research. Firstly, this is a population-based retrospective analysis using the SEER database, which does not include certain important variables, such as preoperative laboratory results and socio-economic status, which are also reported related to the prognosis of patients with bladder malignant tumor [26]. Secondly, although the selection bias is avoided to some extent, a large amount of information is missing in the SEER database, which may have affected the prediction model. Thirdly, this study only considered OS as the primary endpoint and did not include disease-specific survival, which may partly limit the broad application of the results. Fourthly, there are no data available for external validation and due to the nature of retrospective studies; the nomogram needs to be validated for a prospective cohort.

\section{Conclusion}

In this study, we developed and validated a nomogram to predict the OS rate of patients with PBS, and it showed consistent reliability and clinical applicability. The nomogram can assist clinicians in evaluating the risk factors for poor prognosis in patients with PBS and formulating optimal individualized treatment strategies. However, further evaluation in other patient groups is needed to establish the external validity of our findings.

\section{Abbreviations}

OS: Overall survival; PBS: Primary bladder sarcoma; Cl: Confidence interval; SEER: Surveillance, Epidemiology, and End Results; DCA: Decision curve analysis; ROC: Receiver operating characteristics; AUC: The area under the curve; C-index: Concordance index; AJCC: American Joint Committee on Cancer; NRI: Net reclassification improvement; IDI: Integrated discrimination improvement. 


\section{Supplementary Information}

The online version contains supplementary material available at https://doi. org/10.1186/s12894-021-00929-x.

Additional file 1: Figure S1. Nomogram is used to evaluate a 70-year-old patient with T2NOMO and a tumor size of $5 \mathrm{~cm}$. Based on the total score, the survival probability 1 -year, 3 -year, and 5 -year of the patient is $57.5 \%$, $42 \%$, and $34.5 \%$, respectively.

\section{Acknowledgements}

We thank Jie Liu, PhD (Department of Vascular and Endovascular Surgery, Chinese PLA General Hospital) for his helpful review and comments regarding the manuscript. We would like to thank Editage for English language editing.

\section{Authors' contributions}

$X C$ and SL conceived and designed the study, SL contributed to the data collection and analyses of the data. SL drafted and revised the manuscript. SL and $\mathrm{XL}$ prepared figures and tables, $\mathrm{XL}$ and $\mathrm{XC}$ edited the manuscript. All authors read and approved the final manuscript.

\section{Funding}

This study was supported by the Joint plan of key research and development program of Liaoning Province (Grant No. 2020JH 2/10300137), Joint plan of key research and development program of Liaoning Province (Grant No. 2020JH 2/10300148) and the 345 Talent Project of Shengjing Hospital (Grant No. M0716).

\section{Availability of data and materials}

The datasets analyzed during the current study are available in the SEER*Stat software (version 8.3.6, download from https://seer.cancer.gov/data/optio $\mathrm{ns} . \mathrm{html})$. A registration form needs to be completed before using and filter criteria need to be added. The datasets are also available from the corresponding author on reasonable request.

\section{Declarations}

Ethics approval and consent to participate

Since SEER is a publicly available database and all records have been deidentified, no additional ethical approval or informed consent was required after the SEER Research Data Agreement was signed for accessing data. The study was carried out in accordance with the Helsinki Declaration.

\section{Consent for publication}

Not applicable.

\section{Competing interests}

The authors have declared that no conflicts of interest exist.

Received: 22 June 2021 Accepted: 22 November 2021

Published online: 25 November 2021

\section{References}

1. Robinson SP, Farooq A, Laniado M, Motiwala H. The demographic features, clinical outcomes, prognosis and treatment options for patients with sarcomatoid carcinoma of the urinary bladder: a single centre experience. Int Braz J Urol. 2018;44(1):45-52.

2. Guo CC, Majewski T, Zhang L, Yao H, Bondaruk J, Wang Y, et al. Dysregulation of EMT drives the progression to clinically aggressive sarcomatoid bladder cancer. Cell Rep. 2019;27(6):1781-93.

3. Kadouri Y, Ouskri S, El Sayegh H, Benslimane L, Nouini Y. Sarcomatoid carcinoma of the urinary bladder: analysis of five cases and literature review. Pan Afr Med J. 2020:36:369.
4. Dali KM, Kacem A, Ben Rhouma S, Chaker K, Sellami A, Nouira Y. Carcinosarcoma of the urinary bladder with cartilaginous differentiation: about a case report. Urol Case Rep. 2020;28:101053.

5. Ribeiro JGA. Giant leiomyosarcoma of the urinary bladder. J Clin Diagn Res. 2016;10(5):PD14.

6. Alwan MH, Sayed M, Kamal MM. Schistosomiasis and sarcoma of the urinary bladder. Eur Urol. 1988;15(1-2):139.

7. Hendry WF, Parslow JM, Stedronska J. Exploratory scrototomy in 168 azoospermic males. Br J Urol. 1983;55(6):785-91.

8. Mills SE, Fechner RE, Cantrell RW. Aggressive sinonasal lesion resembling normal intestinal mucosa. Am J Surg Pathol. 1982;6(8):803-9.

9. Navon JD, Rahimzadeh M, Wong AK, Carpenter PM, Ahlering TE. Angiosarcoma of the bladder after therapeutic irradiation for prostate cancer. J Urol. 1997;157(4):1359-60.

10. Helpap B. Nonepithelial neoplasms of the urinary bladder. Virchows Arch. 2001:439(4):497-503.

11. Zieschang $H$, Koch $R$, Wirth MP, Froehner M. Leiomyosarcoma of the urinary bladder in adult patients: a systematic review of the literature and meta-analysis. Urol Int. 2019;102(1):96-101.

12. Malla M, Wang JF, Trepeta R, Feng A, Wang J. Sarcomatoid carcinoma of the urinary bladder. Clin Genitourin Cancer. 2016;14(5):366-72.

13. Taylor RE, Busuttil A. Case report: adult rhabdomyosarcoma of bladder, complete response to radiation therapy. J Urol. 1989;142(5):1321.

14. Ahlering TE, Weintraub P, Skinner DG. Management of adult sarcomas of the bladder and prostate. J Urol. 1988;140(6):1397-9.

15. Camp RL, Dolled-Filhart M, Rimm DL. X-tile: a new bio-informatics tool for biomarker assessment and outcome-based cut-point optimization. Clin Cancer Res. 2004;10(21):7252-9.

16. Bray F, Ferlay J, Soerjomataram I, Siegel RL, Torre LA, Jemal A. Global cancer statistics 2018: GLOBOCAN estimates of incidence and mortality worldwide for 36 cancers in 185 countries. CA Cancer J Clin. 2018;68(6):394-424.

17. Alvarez ZR. Bladder sarcoma. Arch Esp Urol. 1947;3(3):217-26.

18. Parekh DJ, Jung C, O'Conner J, Dutta S, Smith ER. Leiomyosarcoma in urinary bladder after cyclophosphamide therapy for retinoblastoma and review of bladder sarcomas. Urology (Ridgewood, NJ). 2002;60(1):164.

19. Rosser CJ, Slaton JW, Izawa JI, Levy LB, Dinney C. Clinical presentation and outcome of high-grade urinary bladder leiomyosarcoma in adults. Urology. 2003;61(6):1151-5.

20. Zieschang $\mathrm{H}$, Koch $\mathrm{R}$, Wirth MP, Froehner M. Leiomyosarcoma of the urinary bladder in adult patients: a systematic review of the literature and meta-analysis. Urol Int. 2018;102(1):96-101.

21. Ro JY, El-Naggar AK, Amin MB, Sahin AA, Ordonez NG, Ayala AG. Pseudosarcomatous fibromyxoid tumor of the urinary bladder and prostate: immunohistochemical, ultrastructural, and DNA flow cytometric analyses of nine cases. Hum Pathol. 1993;24(11):1203-10.

22. Lee TK, Miyamoto H, Osunkoya AO, Guo CC, Weiss SW, Epstein JI. Smooth muscle neoplasms of the urinary bladder: a clinicopathologic study of 51 cases. Am J Surg Pathol. 2010;34(4):502-9.

23. Zhou Z, Wang J, Fang L, Ma J, Guo M. A nomogram for predicting overall survival in patients with E. BMC Musculoskel Dis. 2020;21(1):1-8.

24. Zheng W, Li K, Zhu W, Ding Y, Wu Q, Tang Q, et al. Nomogram prediction of overall survival based on log odds of positive lymph nodes for patients with penile squamous cell carcinoma. Cancer Med-US. 2020;9(15):5425-35

25. Yin K, Luo R, Wei Y, Wang F, Zhang Y, Karlson KJ, et al. Survival outcomes in patients with primary card. J Thorac Cardiovasc Surg. 2020;162(1):107-15.

26. Weiner AB, Keeter M, Manjunath A, Meeks JJ. Discrepancies in staging, treatment, and delays to treatment may explain disparities in bladder cancer outcomes: an update from the National Cancer Data Base (2004-2013). Urol Oncol: Semin Orig Investig. 2018;36(5):237-9.

\section{Publisher's Note}

Springer Nature remains neutral with regard to jurisdictional claims in published maps and institutional affiliations. 